\title{
Formal and Practical Completion of Lagrangian Hybrid Systems
}

\author{
Yizhar Or and Aaron D. Ames
}

\begin{abstract}
This paper presents a method for completing Lagrangian hybrid systems models in a formal manner. That is, given a Lagrangian hybrid system, i.e., a hybrid system that models a mechanical system undergoing impacts, we present a systematic method in which to extend executions of this system past Zeno points by adding an additional domain to the hybrid model. Moreover, by utilizing results that provide sufficient conditions for Zeno behavior and for stability of Zeno equilibria in Lagrangian hybrid systems, we are able to give explicit bounds on the error incurred through the practical simulation of these completed hybrid system models. These ideas are illustrated on a series of examples, and are shown to be consistent with observed reality.
\end{abstract}

\section{INTRODUCTION}

The existence of Zeno behavior-an infinite number of discrete transitions in a finite amount of time-is an intriguing phenomenon which is unique to hybrid systems. When modeling real physical systems as hybrid systems, it is often argued that the Zeno behavior represents an inherent limitation of the hybrid model, since it fails to predict the behavior of the physical system past the Zeno time. This line of reasoning motivates the following question: how does one detect these model shortcomings and overcome them? The answer to this question, like the question itself, is two-fold: one must detect the existence of Zeno behavior and then use the information present in the model to augment it in a way that represents physical reality.

The goal of this paper is to present a method for "completing" hybrid systems that model mechanical systems undergoing impacts: Lagrangian hybrid systems. In particular, we consider a configuration space, a Lagrangian modeling a mechanical system, and a unilateral constraint function that gives the set of admissible configurations for this system. From this data, we obtain a Lagrangian hybrid system. The benefit of studying systems of this form is that they often display Zeno behavior (when an infinite number of collisions occur in a finite amount of time), so they give an ideal class of system in which to gain an intuitive understanding of Zeno behavior, e.g., due to the physical nature of these systems, when they display Zeno behavior it is clear what the system "should" do after reaching a Zeno point.

Using the special structure of Lagrangian hybrid systems, the main observation is that points to which Zeno executions

Y. Or is with the Control and Dynamical Systems Department, California Institute of Technology, Pasadena, CA 91125 izi@cds.caltech.edu.

A. D. Ames is with the Mechanical Engineering Department at Texas A\&M University, College Station, TX 77843. aames@tamu.edu.

Y. Or is supported by Fulbright Postdoctoral Fellowship and Bikura Postdoctoral Scholarship of the Israeli Science Foundation.
converge-Zeno points-must satisfy constraints imposed by the unilateral constraint function. These constraints are holonomic in nature, which implies that after converging to a Zeno point, the hybrid system should switch to a holonomically constrained dynamical system evolving on the zero level set of the unilateral constraint function. Moreover, if the force constraining the dynamical system to the surface becomes zero, there should be a switch back to the original hybrid system. However, while these observations, and the resulting "formal" completion described in this paper are theoretically valuable, they are not practically useful, since in general the exact limit point of a Zeno execution cannot be computed analytically, as it requires computation of infinite number of discrete transitions.

In order to provide a method for completing hybrid systems that is practically applicable, it is first necessary to detect the existence of Zeno behavior. In this work, we utilize conditions for the existence of Zeno behavior and stability of Zeno equilibria which were obtained in [8] and [13]. Due to the constructive nature of the proofs of these results, we are able to leverage them in this paper in order to compute bounds on the errors incurred by a finite truncation of a Zeno execution, which is necessary in practical simulations. These results allow us to propose a method for practically completing hybrid systems such that the truncation errors are guaranteed to satisfy any pre-specified bounds.

There have been many different methods proposed in the literature for dealing with Zeno behavior. The techniques and ideas presented in this paper are motivated by [1], although in that paper the transition from the constrained dynamical system back to the hybrid system was overlooked, sufficient conditions for Zeno behavior were not utilized in the practical completion process, and no formal bounds on truncated Zeno solutions were given. Note that the technique presented here is analogous to the technique proposed in [5] in the context of switched systems, where the solution slides along the switching surface. Another technique that has been proposed in the hybrid systems literature is that of regularization [7], which was applied to specific examples without stating any formal results. In a similar spirit, [11] proposed the notion of generalized solutions, obtained by considering elastic constraint forces and taking the formal limit as the stiffness tends to infinity.

\section{LAGRANGIAN HYBRID SYSTEMS}

In this section, we introduce the notion of a hybrid Lagrangian, the associated Lagrangian hybrid system, and discuss Zeno behavior and the corresponding notion of Zeno equilibria in systems of this form. Hybrid systems of this 


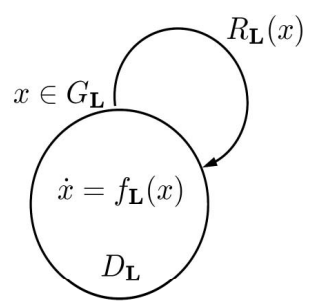

Fig. 1. A graphical representation of a simple hybrid system.

form have been studied in the context of Zeno behavior see [1], [8], and were also formulated as linear complementarity systems in [3] and [16].

We begin this section by reviewing the notion of a simple hybrid system (A graphical representation of a simple hybrid system can be seen in Fig. 1).

Definition 1: A simple hybrid system is a tuple:

$$
\mathscr{H}=(D, G, R, f),
$$

where

- $D$ is a smooth manifold called the domain,

- $G$ is an embedded submanifold of $D$ called the guard,

- $R$ is a smooth map $R: G \rightarrow D$ called the reset map,

- $f$ is a smooth vector field on the manifold $D$.

This paper focuses on simple hybrid systems, having a single domain, guard, reset map, and vector field. A general hybrid system (see [9] and [17]), which is not discussed here, consists of a collection of domains, guards, reset maps and vector fields as indexed by an oriented graph.

Hybrid executions. A hybrid execution ${ }^{1}$ of a simple hybrid system $\mathscr{H}$ is a tuple $\chi^{\mathscr{H}}=(\Lambda, \mathcal{J}, \mathcal{C})$, where

- $\Lambda=\{0,1,2, \ldots\} \subseteq \mathbb{N}$ is an indexing set.

- $\mathcal{J}=\left\{I_{i}\right\}_{i \in \Lambda}$ is a hybrid interval where $I_{i}=\left[\tau_{i}, \tau_{i+1}\right]$ if $i, i+1 \in \Lambda$ and $I_{N-1}=\left[\tau_{N-1}, \tau_{N}\right]$ or $\left[\tau_{N-1}, \tau_{N}\right)$ or $\left[\tau_{N-1}, \infty\right)$ if $|\Lambda|=N, N$ finite. Here, $\tau_{i}, \tau_{i+1}, \tau_{N} \in \mathbb{R}$ and $\tau_{i} \leq \tau_{i+1}$.

- $\mathcal{C}=\left\{c_{i}\right\}_{i \in \Lambda}$ is a collection of integral curves of $f$, i.e., $\dot{c}_{i}(t)=f\left(c_{i}(t)\right)$ for $t \in I_{i}, i \in \Lambda$,

and the following conditions hold for every $i, i+1 \in \Lambda$ :

$$
\begin{aligned}
& c_{i}\left(\tau_{i+1}\right) \in G, \\
& R\left(c_{i}\left(\tau_{i+1}\right)\right)=c_{i+1}\left(\tau_{i+1}\right), \\
& \tau_{i+1}=\min \left\{t \in I_{i}: c_{i}(t) \in G\right\} .
\end{aligned}
$$

The initial condition for the hybrid execution is $c_{0}\left(\tau_{0}\right)$.

Lagrangians. Let $Q$ be the $n$-dimensional configuration space for a mechanical system (assumed to be a smooth manifold) and $T Q$ the tangent bundle of $Q$. In this paper, we will consider Lagrangians, $L: T Q \rightarrow \mathbb{R}$, describing mechanical systems, which take the form

$$
L(q, \dot{q})=\frac{1}{2} \dot{q}^{T} M(q) \dot{q}-V(q)
$$

where $M(q)$ is the (positive definite) inertial matrix, $\frac{1}{2} \dot{q}^{T} M(q) \dot{q}$ is the kinetic energy and $V(q)$ is the potential

\footnotetext{
${ }^{1}$ Note that we refer to an execution of a hybrid system as a "hybrid execution" in order to differentiate it from other types of executions that will be considered in this paper.
}

energy. In this case, the Euler-Lagrange equations yield the (unconstrained) equations of motion for the system:

$$
M(q) \ddot{q}+C(q, \dot{q}) \dot{q}+N(q)=0
$$

where $C(q, \dot{q})$ is the Coriolis matrix (cf. [12]) and $N(q)=$ $\frac{\partial V}{\partial q}(q)$. Setting $x=(q, \dot{q})$, the Lagrangian vector field, $f_{L}$, associated to $L$ takes the familiar form:

$$
\dot{x}=f_{L}(x)=\left(\begin{array}{c}
\dot{q} \\
M(q)^{-1}(-C(q, \dot{q}) \dot{q}-N(q))
\end{array}\right) .
$$

This process of associating a dynamical system to a Lagrangian will be mirrored in the setting of hybrid systems. First, we introduce the notion of a hybrid Lagrangian.

Definition 2: A simple hybrid Lagrangian is defined to be a tuple

$$
\mathbf{L}=(Q, L, h)
$$

where

- $Q$ is the configuration space,

- $L: T Q \rightarrow \mathbb{R}$ is a hyperregular Lagrangian,

- $h: Q \rightarrow \mathbb{R}$ provides a unilateral constraint on the configuration space; we assume that the zero level set $h^{-1}(0)$ is a smooth manifold.

Simple Lagrangian hybrid systems. For a Lagrangian (1), there is an associated dynamical system (3). Similarly, given a hybrid Lagrangian $\mathbf{L}=(Q, L, h)$ the simple Lagrangian hybrid system (SLHS) associated to $\mathbf{L}$ is the simple hybrid system:

$$
\mathscr{H}_{\mathbf{L}}=\left(D_{\mathbf{L}}, G_{\mathbf{L}}, R_{\mathbf{L}}, f_{\mathbf{L}}\right)
$$

First, we define

$$
\begin{aligned}
& D_{\mathbf{L}}=\{(q, \dot{q}) \in T Q: h(q) \geq 0\}, \\
& G_{\mathbf{L}}=\{(q, \dot{q}) \in T Q: h(q)=0 \text { and } d h(q) \dot{q} \leq 0\},
\end{aligned}
$$

where $d h(q)=\left(\frac{\partial h}{\partial q}(q)\right)^{T}=\left(\begin{array}{lll}\frac{\partial h}{\partial q_{1}}(q) & \cdots & \frac{\partial h}{\partial q_{n}}(q)\end{array}\right)$.

In this paper, we adopt the reset map:

$$
R_{\mathbf{L}}(q, \dot{q})=\left(q, P_{\mathbf{L}}(q, \dot{q})\right)
$$

which based on the impact equation ([2])

$$
P_{\mathbf{L}}(q, \dot{q})=\dot{q}-(1+e) \frac{d h(q) \dot{q}}{d h(q) M(q)^{-1} d h(q)^{T}} M(q)^{-1} d h(q)^{T},
$$

where $0 \leq e \leq 1$ is the coefficient of restitution, which is a measure of the energy dissipated through impact. This reset map corresponds to rigid-body collision law under the assumption of frictionless impact [2]. Examples of more complicated collision laws that account for friction can be found in [2] and [4]. Finally, $f_{\mathbf{L}}=f_{L}$ is the Lagrangian vector field associated to $L$ in (3).

Zeno behavior. A hybrid execution $\chi^{\mathscr{H}}$ is Zeno if $\Lambda=\mathbb{N}$ and $\lim _{i \rightarrow \infty} \tau_{i}=\tau_{\infty}<\infty$. Here $\tau_{\infty}$ is called the Zeno time. If $\chi^{\mathscr{H}_{\mathrm{L}}}$ is a Zeno execution of a Lagrangian hybrid system $\mathscr{H}_{\mathbf{L}}$, then its Zeno point is defined to be

$$
c_{\infty}=\left(q_{\infty}, \dot{q}_{\infty}\right)=\lim _{i \rightarrow \infty} c_{i}\left(\tau_{i}\right)=\lim _{i \rightarrow \infty}\left(q_{i}\left(\tau_{i}\right), \dot{q}_{i}\left(\tau_{i}\right)\right) .
$$




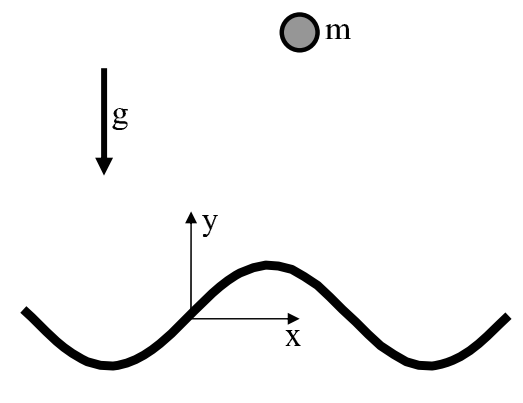

(a)

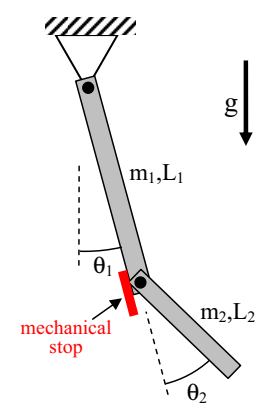

(b)
Fig. 2. (a) The ball on a sinusoidal surface (b) The double pendulum

These limit points are intricately related to a type of equilibrium points that are unique to hybrid systems: Zeno equilibria.

Definition 3: A Zeno equilibrium point of a simple hybrid system $\mathscr{H}$ is a point $x^{*} \in G$ such that

- $R\left(x^{*}\right)=x^{*}$,

- $f\left(x^{*}\right) \neq 0$.

Zeno equilibria. If $\mathscr{H}_{\mathbf{L}}$ is a Lagrangian hybrid system, then due to the special form of these systems we find that the point $\left(q^{*}, \dot{q}^{*}\right)$ is a Zeno equilibria iff $\dot{q}^{*}=P_{\mathbf{L}}\left(q^{*}, \dot{q}^{*}\right)$, with $P_{\mathbf{L}}$ given in (4). In particular, the special form of $P_{\mathbf{L}}$ implies that this holds iff $d h\left(q^{*}\right) \dot{q}^{*}=0$. Therefore the set of all Zeno equilibria for a Lagrangian hybrid system is given by the hypersurface in $G_{\mathbf{L}}$ :

$$
Z=\left\{(q, \dot{q}) \in G_{\mathbf{L}}: d h(q) \dot{q}=0\right\} .
$$

Note that if $\operatorname{dim}(Q)>1$, the Zeno equilibria in Lagrangian hybrid systems are always non-isolated (see [8])— this motivates the study of such equilibria.

Example 1 (Ball): The first running example of this paper is a planar model of a ball bouncing on a sinusoidal surface (cf. Fig. 2(a)). The ball is modeled as a point mass $m$. In this case

$$
\mathbf{B}=\left(Q_{\mathbf{B}}, L_{\mathbf{B}}, h_{\mathbf{B}}\right),
$$

where $Q_{\mathrm{B}}=\mathbb{R}^{2}$, and the configuration is the position of the ball $q=(x, y)$,

$$
L_{\mathbf{B}}(x, \dot{x})=\frac{1}{2} m\|\dot{q}\|^{2}-m g y .
$$

Finally, the ball's configuration is unilaterally constrained by the constraint function $h_{\mathbf{B}}(q)=y-\sin (x) \geq 0$. So, for this example, there are trivial dynamics and a nontrivial constraint function.

Note that from the hybrid Lagrangian $\mathbf{B}=\left(Q_{\mathbf{B}}, L_{\mathbf{B}}, h_{\mathbf{B}}\right)$ we obtain a hybrid system $\mathscr{H}_{\mathbf{B}}=\left(D_{\mathbf{B}}, G_{\mathbf{B}}, R_{\mathbf{B}}, f_{\mathbf{B}}\right)$. The set of Zeno equilibria for this hybrid system are given by:

$$
Z=\left\{(x, y, \dot{x}, \dot{y}) \in G_{\mathbf{B}}: \dot{y}-\dot{x} \cos (x)=0\right\} .
$$

Physically, this set corresponds to states at which the ball slides along the sinusoidal surface.

Example 2 (Double Pendulum): Our second running example is a constrained double pendulum with a mechanical stop (cf. Fig. 2(b)). The double pendulum consists of two rigid links of masses $m_{1}, m_{2}$, lengths $L_{1}, L_{2}$, and uniform mass distribution, which are attached by passive joints, while a mechanical stop dictates the range of motion of the second link. The example serves as a simplified model of a leg with a passive knee and a mechanical stop, which is widely investigated in the robotics literature in the context of passive dynamics of bipedal walkers (cf. [10] and [15]). In this case

$$
\mathbf{P}=\left(Q_{\mathbf{P}}, L_{\mathbf{P}}, h_{\mathbf{P}}\right),
$$

where $Q_{\mathbf{P}}=\mathbb{S}^{1} \times \mathbb{S}^{1}, q=\left(\theta_{1}, \theta_{2}\right)$, and

$$
\begin{gathered}
L_{\mathbf{P}}(q, \dot{q})=\frac{1}{2} \dot{q}^{T} M(q) \dot{q}+\left(\frac{1}{2} m_{1} L_{1}+m_{2} L_{1}\right) g \cos \theta_{1} \\
+\frac{1}{2} m_{2} L_{2} g \cos \left(\theta_{1}+\theta_{2}\right),
\end{gathered}
$$

with the elements of the $2 \times 2$ inertia matrix $M(q)$ given by

$$
\begin{aligned}
& M_{11}=m_{1} L_{1}^{2} / 3+m_{2}\left(L_{1}^{2}+L_{2}^{2} / 3+L_{1} L_{2} \cos \theta_{2}\right) \\
& M_{12}=M_{21}=m_{2}\left(3 L_{1} L_{2} \cos \theta_{2}+2 L_{2}^{2}\right) / 6 \\
& M_{22}=m_{2} L_{2}^{2} / 3 .
\end{aligned}
$$

Finally, the constraint that represents the mechanical stop is given by $h_{\mathbf{P}}(q)=\theta_{2} \geq 0$. So, for this example, there are nontrivial dynamics and a trivial constraint function.

Note that from the hybrid Lagrangian $\mathbf{P}=\left(Q_{\mathbf{P}}, L_{\mathbf{P}}, h_{\mathbf{P}}\right)$ we obtain a hybrid system $\mathscr{H}_{\mathbf{P}}=\left(D_{\mathbf{P}}, G_{\mathbf{P}}, R_{\mathbf{P}}, f_{\mathbf{P}}\right)$. The set of Zeno equilibria for this hybrid system are given by:

$$
Z=\left\{\left(\theta_{1}, \theta_{2}, \dot{\theta}_{1}, \dot{\theta}_{2}\right) \in D_{\mathbf{P}}: \theta_{2}=0, \dot{\theta}_{2}=0\right\} .
$$

That is, the set of Zeno equilibria are the set of points where the lower link (i.e. the "knee") is locked.

\section{The Completed LagRangian Hybrid System}

In this section, we present a method for carrying executions of Lagrangian hybrid systems beyond Zeno equilibria, i.e., we formally define a "completed" hybrid system.

Overview of completion process. The motivation for completing hybrid system models is based upon the fact that despite the name "Zeno equilibrium," a Zeno point $c_{\infty}$ is not a physical equilibrium point, since it satisfies $f_{\mathbf{L}}\left(c_{\infty}\right) \neq 0$, and involves nonzero velocity. It was postulated [1] that after the Zeno time, the system should switch to a holonomically constrained dynamical system. Note that this postulation is essentially a modeling paradigm, and as such, its correctness cannot be mathematically proven. However, we argue that this concept correctly captures the physical behavior of mechanical systems with unilateral constraints, modeled here as Lagrangian hybrid systems.

The main observation of [1] is that a Zeno execution of a Lagrangian Hybrid System converges to a limit point $\left(q_{\infty}, \dot{q}_{\infty}\right)$ that satisfies $h\left(q_{\infty}\right)=0$ and $d h\left(q_{\infty}\right) \dot{q}_{\infty}=0$. This limit point lies on the surface $h^{-1}(0)=\{q \in Q: h(q)=0\}$, and its velocity vector is tangent to this surface. Since in Lagrangian hybrid systems the unilateral constraint $h(q) \geq 0$ usually represents a mechanical contact, it is hypothesized in [1] that once such a contact is re-established via a Zeno execution, it is then maintained by a constraining force. This behavior is captured by the formulation of a holonomically constrained dynamical system whose trajectories 
are constrained to the surface $h^{-1}(0)$, where the constraint is maintained by a Lagrange multiplier $\lambda$, representing the physical contact force.

An important fact that was overlooked in [1] is that, in such systems, a contact force is often also constrained to be non-negative, thus eliminating tension or adhesion forces. Under this assumption, our completed model suggests that at a zero-crossing event of the constraint force, the constrained system switches back to the hybrid system. Physically, this event corresponds to contact breakage and separation.

Constrained dynamical systems. We now define the holonomically constrained dynamical system $\mathscr{D}_{\mathbf{L}}$ associated with the hybrid Lagrangian $\mathbf{L}$. For such systems, the constrained equations of motion can be obtained from the equations of motion for the unconstrained system (2), and are given by (cf. [12]):

$$
M(q) \ddot{q}+C(q, \dot{q}) \dot{q}+N(q)=d h(q)^{T} \lambda,
$$

where $\lambda$ is the Lagrange multiplier which represents the contact force. Differentiating the constraint $h(q(t))=0$ twice with respect to time and substituting the solution for $\ddot{q}$ in (5), the solution for the constraint force $\lambda$ is obtained as follows:

$$
\begin{aligned}
& \lambda(q, \dot{q})=\left(d h(q) M(q)^{-1} d h(q)^{T}\right)^{-1} \\
& \quad\left(d h(q) M(q)^{-1}(C(q, \dot{q}) \dot{q}+N(q))-\dot{q}^{T} H(q) \dot{q}\right),
\end{aligned}
$$

where $H(q)$ is the Hessian of $h$ at $q$. From the constrained equations of motion, for $x=(q, \dot{q})$, we get the vector field

$\dot{x}=f_{L}^{\lambda}(x)=\left(\begin{array}{c}\dot{q} \\ -M(q)^{-1}\left(C(q, \dot{q}) \dot{q}+N(q)-d h(q)^{T} \lambda(q, \dot{q})\right)\end{array}\right)$

Note that $f_{L}^{\lambda}$ defines a vector field on the manifold $\left.T Q\right|_{h^{-1}(0)}$, from which we obtain the dynamical system $\mathscr{D}_{\mathbf{L}}=\left(\left.T Q\right|_{h^{-1}(0)}, f_{L}^{\lambda}\right)$. For this dynamical system, $q(t)$ slides along the surface $h^{-1}(0)$ as long as the constraint force $\lambda$ is positive.

A constrained execution $\tilde{\chi}$ of $\mathscr{D}_{\mathbf{L}}$ is a pair $(\tilde{I}, \tilde{c})$ where $\tilde{I}=\left[\tilde{t}_{0}, \tilde{t}_{f}\right] \subset \mathbb{R}$ if $\tilde{t}_{f}$ is finite and $\tilde{I}=\left[\tilde{t}_{0}, \tilde{t}_{f}\right) \subset \mathbb{R}$ if $\tilde{t}_{f}=\infty$ and $\tilde{c}: I \rightarrow T Q$, with $\tilde{c}(t)=(q(t), \dot{q}(t))$ a solution of (5) that satisfies the following properties:

$$
\begin{aligned}
& h\left(q\left(\tilde{t}_{0}\right)\right)=0, \\
& d h\left(q\left(\tilde{t}_{0}\right)\right) \dot{q}\left(\tilde{t}_{0}\right)=0, \\
& \lambda\left(q\left(\tilde{t}_{0}\right), \dot{q}\left(\tilde{t}_{0}\right)\right)>0, \\
& \tilde{t}_{f}=\min \{t \in \tilde{I}: \lambda(q(t), \dot{q}(t))=0\} .
\end{aligned}
$$

Using the notation and concepts introduced thus far, we introduce the notion of a completed hybrid system.

Definition 4: If $\mathbf{L}$ is a simple hybrid Lagrangian and $\mathscr{H}_{\mathbf{L}}$ the corresponding Lagrangian hybrid system, the corresponding completed Lagrangian hybrid system ${ }^{2}$ is defined to be:

$$
\overline{\mathscr{H}}_{\mathbf{L}}:=\left\{\begin{array}{c}
\mathscr{D}_{\mathbf{L}} \\
\mathscr{H}_{\mathbf{L}}
\end{array} \quad \text { if } \quad h(q)=0, \begin{array}{c}
\operatorname{dh}(q) \dot{q}=0, \quad \lambda(q, \dot{q})>0 \\
\text { otherwise. }
\end{array}\right.
$$

\footnotetext{
${ }^{2}$ As was orginally pointed out in [1], this terminology (and notation) is borrowed from topology, where a metric space can be completed to ensure that "limits exist."
}

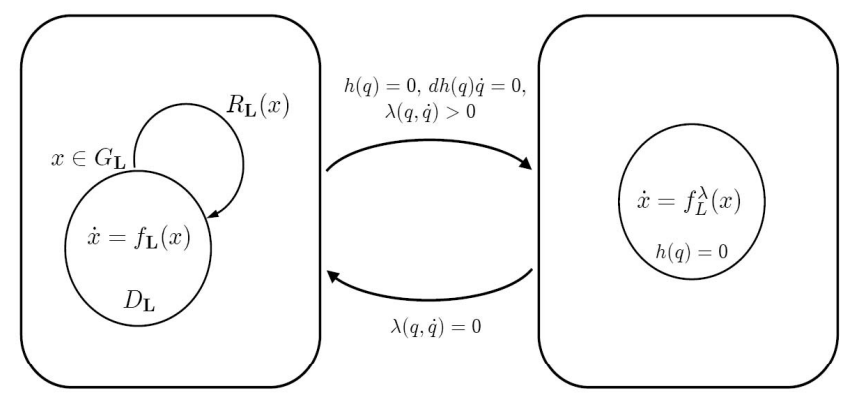

Fig. 3. A graphical representation of a completed hybrid system.

Remarks. First, note that the only way for the transition to be made from the hybrid system $\mathscr{H}_{\mathbf{L}}$ to the constrained system $\mathscr{D}_{\mathbf{L}}$ is if a specific Zeno execution reaches its Zeno point. Second, a transition from $\mathscr{D}_{\mathbf{L}}$ to $\mathscr{H}_{\mathbf{L}}$ occurs when the constraint force $\lambda$ crosses zero. A graphical representation of the completed system $\overline{\mathscr{H}}_{\mathbf{L}}$ is illustrated in Fig. 3. Finally, let $\ddot{h}(q, \dot{q})$ be the acceleration of $h(q(t))$ along trajectories of the unconstrained dynamics (2), given by ([13]):

$$
\ddot{h}(q, \dot{q})=\dot{q}^{T} H(q) \dot{q}-d h(q) M(q)^{-1}(C(q, \dot{q}) \dot{q}+N(q)) .
$$

The definitions of $\ddot{h}(q, \dot{q})$ in (8) and $\lambda(q, \dot{q})$ in (6) imply that these two quantities are in complementarity relations, that is, while the solution slides along the surface $h^{-1}(0)$, either $\ddot{h}=0$ and $\lambda>0$, corresponding to maintaining constrained motion, or $\ddot{h}>0$ and $\lambda=0$, corresponding to leaving the constraint surface and switching back to the hybrid system. Thus, the definition of the completed hybrid system is consistent ${ }^{3}$.

The completed execution Having introduced the completed hybrid system, we now introduce the semantics of solutions of systems of this form, which is captured by the notion of completed execution of a completed hybrid system.

Definition 5: Given a simple hybrid Lagrangian $\mathbf{L}$ and the associated completed system $\overline{\mathscr{H}}_{\mathbf{L}}$, a completed execution $\bar{\chi}$ is a (possibly infinite) ordered sequence of alternating hybrid and constrained executions

$$
\bar{\chi}=\left\{\chi^{(1)}, \tilde{\chi}^{(2)}, \chi^{(3)}, \tilde{\chi}^{(4)}, \ldots\right\}
$$

that satisfies the following conditions:

(i) For each pair $\chi^{(i)}$ and $\tilde{\chi}^{(i+1)}$,

$$
\tau_{\infty}^{(i)}=\tilde{t}_{0}^{(i+1)} \text { and } c_{\infty}^{(i)}=\tilde{c}_{0}^{(i+1)}\left(\tilde{t}_{0}^{(i+1)}\right) .
$$

(ii) For each pair $\tilde{\chi}^{(i)}$ and $\chi^{(i+1)}$,

$$
\tilde{t}_{f}^{(i)}=\tau_{0}^{(i+1)} \text { and } \tilde{c}^{(i)}\left(\tilde{t}_{f}^{(i)}\right)=c_{0}^{(i+1)}\left(\tau_{0}^{(i+1)}\right) .
$$

where the superscript $(i)$ denotes values corresponding to the $i$-th execution in $\bar{\chi}$, and $\tau_{\infty}^{(i)}, c_{\infty}^{(i)}$ denote the Zeno time and Zeno point associated with the $i$-th hybrid execution $\chi^{(i)}$.

Note that the first element of $\bar{\chi}$ can also be a constrained execution $\tilde{\chi}^{(1)}$, as long as the overall initial conditions satisfy conditions (i)-(iii) in (7). Note, too, that $\bar{\chi}$ can also consist of a finite number of executions, where the last execution,

\footnotetext{
${ }^{3}$ Note that adding Coulomb's friction to the constrained motion leads to possible inconsistencies of the solution [2].
} 
which can be either a constrained execution or a non-Zeno hybrid execution, extends to infinite time.

\section{Practical Completion and Zeno Stability}

In this section, we discuss some of the practical difficulties arising in numerical simulation of completed hybrid systems near Zeno points. Motivated by these difficulties, we review some relevant results regarding Zeno behavior and stability of Zeno equilibria.

Observations. An important observation is that the notion of completed hybrid system described in Section III is not practically useful; to be successfully implemented, one would need exact knowledge of Zeno executions. Since such exact knowledge cannot be assumed as it requires computing an infinite number of discrete transitions, one must be prepared to present a practical method for completing hybrid systems that will be amenable to simulation. That is, any software implementation of the numerical simulation of Zeno executions will necessarily involve a finite truncation of the infinite sequence of discrete transitions. This leads to the conclusion that a notion of completing hybrid systems practically must be introduced - one that can handle errors incurred by the finite truncation of Zeno executions.

The practical realities of simulating Zeno executions pose two problems regarding the reliability of the numerical approximations, that must be addressed. Firstly, one needs to guarantee that an approximation of a Zeno execution actually detects the true Zeno behavior of the exact execution, and not just an aliasing effect resulting from truncations. Secondly, recall that as part of the completion process, the numerical simulation would need to generate an approximate Zeno point which then serves as an initial condition for the next phase of constrained dynamics. Therefore, in order to generate a reliable simulation, one needs to guarantee that the approximated Zeno point lies in an arbitrarily close neighborhood of the exact Zeno limit point (which cannot be computed analytically), that is, the approximation error should satisfy a pre-specified bound. These two problems will be addressed in this paper through the utilization of relevant results regarding sufficient conditions for Zeno behavior and conditions for stability of Zeno equilibria. Therefore, before discussing how to practically complete hybrid systems, we will first review these relevant results.

Sufficient conditions for Zeno behavior. In order to address the problem of reliably detecting Zeno executions, we now present sufficient conditions for existence of Zeno executions in the vicinity of a Zeno equilibrium point of a simple Lagrangian hybrid system. These conditions are based on evaluating the acceleration $\ddot{h}(q, \dot{q})$ (Eq. (8)) at the Zeno equilibrium point, as summarized in the following theorem.

Theorem 1 ([8]): Let $\mathscr{H}_{\mathrm{L}}$ be a simple Lagrangian hybrid system and Let $x^{*}=\left(q^{*}, \dot{q}^{*}\right)$ be a Zeno equilibrium point of $\mathscr{H}_{\mathbf{L}}$. Then if $e<1$ and $\ddot{h}\left(q^{*}, \dot{q}^{*}\right)<0$, there exists a neighborhood $W \subset D_{\mathbf{L}}$ of $x^{*}$ such that for every $x_{0} \in W$, there is a unique Zeno execution $\chi^{\mathscr{H}_{\mathbf{L}}}$ with $c_{0}\left(\tau_{0}\right)=x_{0}$.
This theorem provides a way for detecting candidate Zeno limit points, such that truncating an execution in their vicinity and switching to a constrained system preserve the qualitative behavior of the exact solution. However, the theorem does not provide any quantitative measure of how close to the limit point one should truncate, and how large the resulting approximation error is. This motivates the definition of stability of Zeno equilibria, given as follows:

Definition 6: Let $x^{*}=\left(q^{*}, \dot{q}^{*}\right)$ be a Zeno equilibrium point of a simple Lagrangian hybrid system $\mathscr{H}_{\mathbf{L}}$. Then $x^{*}$ is defined as bounded-time locally stable (BTLS) if for each open neighborhood $U \subseteq T Q$ of $x^{*}$ and $\epsilon_{t}>0$, there exists another open neighborhood $W$ of $x^{*}$, such that for every initial condition $c_{0}\left(\tau_{0}\right) \in W \cap D_{\mathbf{L}}$, the corresponding hybrid execution $\chi^{\mathscr{H}_{\mathbf{L}}}$ is Zeno, and satisfies $c_{i}(t) \in U$ for all $t \in I_{i}$ and $i \in \Lambda$, while its Zeno time satisfies $\tau_{\infty}-\tau_{0}<\epsilon_{t}$.

The definition is equivalent to the notion of uniform Zeno stability defined in [6]. The following theorem establishes conditions for the BTLS of Zeno equilibria of SLHS.

Theorem 2 ([13]): Let $x^{*}=\left(q^{*}, \dot{q}^{*}\right)$ be a Zeno equilibrium point of a simple Lagrangian hybrid system $\mathscr{H}_{\mathbf{L}}$. Then the following two conditions hold:

\section{(i) If $e<1$ and $\ddot{h}\left(q^{*}, \dot{q}^{*}\right)<0$, then $x^{*}$ is BTLS \\ (ii) If $\ddot{h}\left(q^{*}, \dot{q}^{*}\right)>0$, then $x^{*}$ is not BTLS}

This theorem implies that the conditions given in Theorem 1 are also sufficient for bounded-time local stability. More importantly, the proof of the theorem, which appears in [13], provides an explicit construction of the neighborhood $W$ for a given neighborhood $U$, which is fundamental to the practical completion of hybrid systems.

Explicit construction of neighborhoods. We conclude this section by reviewing the derivation of explicit expressions for two intermediate neighborhoods associated with a given $U$ in Theorem 2, which will prove useful in our context of practical completion with bounded error. For the sake of concreteness and simplicity, we use a local coordinate chart for small neighborhoods of $x^{*}$. Therefore, we can identify both $q$ and $\dot{q}$ with elements of $\mathbb{R}^{n}$, and use the induced Euclidean norm $\|\cdot\|$ to define neighborhoods of $x^{*}$ as

$N\left(\epsilon_{q}, \epsilon_{v}\right)=\left\{(q, \dot{q}) \in D_{\mathbf{L}}:\left\|q-q^{*}\right\|<\epsilon_{q}\right.$ and $\left.\left\|\dot{q}-\dot{q}^{*}\right\|<\epsilon_{v}\right\}$. Using this notation, for a given $U$ there exist $\epsilon_{q}$ and $\epsilon_{v}$ such that $U \subseteq N\left(\epsilon_{q}, \epsilon_{v}\right)$. Thus, we assume that $U$ is initially in the form $U=N\left(\epsilon_{q}, \epsilon_{v}\right)$. We now review two lemmas which were used to prove Theorem 2 in [13]. The first lemma defines an intermediate neighborhood $V \in U$, such that any execution that stays within $V$ at all times is guaranteed to be Zeno.

Lemma 1 ([13]): Let $x^{*}=\left(q^{*}, \dot{q}^{*}\right)$ be a Zeno equilibrium point of a simple Lagrangian hybrid system such that $\ddot{h}\left(q^{*}, \dot{q}^{*}\right)<0$ and $e<1$. Let $U=N\left(\epsilon_{q}, \epsilon_{v}\right)$ be a given neighborhood of $x^{*}$, and define the neighborhood $V=N\left(\epsilon_{q}^{\prime}, \epsilon_{v}^{\prime}\right)$, where $\epsilon_{q}^{\prime}<\epsilon_{q}$ and $\epsilon_{v}^{\prime}<\epsilon_{v}$ are chosen as to satisfy the conditions:

$$
a_{\max }>a_{\min }>0 \quad \text { and } \quad e \frac{a_{\max }}{a_{\min }}<1,
$$




$$
\text { where } \begin{aligned}
a_{\min } & =-\max _{(q, \dot{q}) \in V} \ddot{h}(q, \dot{q}), \\
a_{\max } & =-\min _{(q, \dot{q}) \in V} \ddot{h}(q, \dot{q}) .
\end{aligned}
$$

Then any execution $\chi^{\mathscr{H}_{\mathbf{L}}}$ such that $c_{i}(t) \in V$ for all $t \in I_{i}$ and $i \in \Lambda$, is Zeno.

The second lemma defines a neighborhood $W^{\prime} \subset G_{\mathbf{L}} \cap V$, which lies on the guard $G_{\mathbf{L}}$, such that any execution whose first collision point $c_{0}\left(\tau_{1}\right)$ lies within $W^{\prime}$ is guaranteed to stay within $V$. First, we define the following scalars:

$$
\begin{aligned}
e^{\prime} & =e \frac{a_{\max }}{a_{\min }} \\
e^{\prime \prime} & =e \sqrt{\frac{a_{\max }}{a_{\min }}} \\
\beta & =\left\|\dot{q}^{*}\right\|+\epsilon_{v}^{\prime} \\
\eta & =\max _{(q, \dot{q}) \in V} \frac{\left\|M^{-1}(q) d h(q)^{T}\right\|}{d h(q) M(q) d h(q)^{T}} \\
\zeta & =\max _{(q, \dot{q}) \in V}\left\|M^{-1}(q)(C(q, \dot{q}) \dot{q}+N(q))\right\| .
\end{aligned}
$$

Using these definitions, the lemma is as follows.

Lemma 2 ([13]): Let $x^{*}=\left(q^{*}, \dot{q}^{*}\right)$ be a Zeno equilibrium point of a simple Lagrangian hybrid system $\mathscr{H}_{\mathbf{L}}$ such that $\ddot{h}\left(q^{*}, \dot{q}^{*}\right)<0$ and $e<1$, and let $V=N\left(\epsilon_{q}^{\prime}, \epsilon_{v}^{\prime}\right)$ be a neighborhood of $x^{*}$ that satisfies (9). For a given $\epsilon_{t}>0$, define the neighborhood $W^{\prime}$ as

$$
\begin{array}{r}
W^{\prime}=\left\{(q, \dot{q}) \in T Q: \quad h(q)=0,\left\|q-q^{*}\right\| \leq \delta_{q}^{\prime},\right. \\
\left.\left\|\dot{q}-\dot{q}^{*}\right\| \leq \delta_{v}^{\prime}, \text { and } d h(q) \dot{q}<-v_{1 \max }<0\right\},
\end{array}
$$

such that $\delta_{q}^{\prime}, \delta_{v}^{\prime}$ and $v_{1 \max }$ satisfy the conditions

$$
\begin{aligned}
\delta_{q}^{\prime}<\epsilon_{q}^{\prime}, \delta_{v}^{\prime} & <\epsilon_{v}^{\prime}, \quad v_{1 \max }<\min \left\{c_{1}, c_{2}, c_{3}\right\} \\
\text { where } c_{1} & =\frac{a_{\min }\left(1-e^{\prime}\right)}{2 e} \epsilon_{t} \\
c_{2} & =\frac{a_{\min }\left(1-e^{\prime}\right)}{2 e \beta}\left(\epsilon_{q}^{\prime}-\delta_{q}^{\prime}\right) \\
c_{3} & =\left(\epsilon_{v}^{\prime}-\delta_{v}^{\prime}\right) /\left(\frac{(1+e) \eta}{1-e^{\prime \prime}}+\frac{2 e \zeta}{a_{\min }\left(1-e^{\prime}\right)}\right) .
\end{aligned}
$$

Then each execution $\chi^{\mathscr{H}_{\mathbf{L}}}$ such that $c_{0}\left(\tau_{1}\right) \in W^{\prime}$ is Zeno, and satisfies $c_{i}(t) \in V$ for all $t \in I_{i}$ and $i \geq 1$. Moreover, the corresponding Zeno time satisfies $\tau_{\infty}-\tau_{1}<\epsilon_{t}$.

These results will be utilized in the next section for constructing a reliable approximation for the completed execution, with guaranteed error bounds.

\section{Practical Completion of Hybrid Systems}

In this section we describe the procedure of computing a reliable approximation for the execution of a completed hybrid system, with guaranteed bounds on the approximation error, based on the results of Lemmas 1 and 2 regarding stability of Zeno equilibria. This is a key result of this paper.

Overview. The main idea behind the reliable approximation algorithm is now outlined. First, a hybrid execution is simulated, until it reaches a collision at some time $\tau_{k}$, with the state $\left(q\left(\tau_{k}\right), \dot{q}\left(\tau_{k}\right)\right)$ satisfying certain conditions, called the reliable truncation conditions. At that point, the hybrid execution is truncated, and the algorithm applies a re-initialization map $R^{*}$ that maps the state $(q, \dot{q})$ at the time of truncation into a Zeno equilibrium point $\left(q^{*}, \dot{q}^{*}\right)$. The algorithm then switches to simulating the constrained dynamics (5), with the initial conditions given by $\left(q^{*}, \dot{q}^{*}\right)$.

We now define the re-initialization map $R^{*}$, and then define the reliable truncation conditions. The re-initialization map $R^{*}: D_{\mathbf{L}} \rightarrow Z$ is given by $R^{*}(q, \dot{q})=\left(q^{*}, \dot{q}^{*}\right)$, where

$$
q^{*}=q \quad \text { and } \quad \dot{q}^{*}=\dot{q}-\frac{d h(q) \dot{q}}{\|d h(q)\|^{2}} d h(q)^{T} .
$$

Note that since $R^{*}$ is applied at a collision time, $q$ already satisfies $h(q)=0$. Moreover, under the map $R^{*}$, the velocity $\dot{q}$ is projected orthogonally onto the plane $d h(q) \dot{q}=0$. Thus, it is clear that $\left(q^{*}, \dot{q}^{*}\right)$ is actually a Zeno equilibrium point.

We now define the reliable truncation conditions, depending on the given bounds $\epsilon_{q}, \epsilon_{v}$, and $\epsilon_{t}$, which are the desired bounds on the position, velocity, and time errors, respectively, caused by the truncation.

Definition 7: Let $(q, \dot{q})$ be a state of $\mathscr{H}_{\mathbf{L}}$ such that $h(q)=$ 0 and $d h(q) \dot{q}<0$, and denote $\left(q^{*}, \dot{q}^{*}\right)=R^{*}(q, \dot{q})$. Then for given $\epsilon_{q}, \epsilon_{v}$, and $\epsilon_{t}$, the reliable truncation conditions for $(q, \dot{q})$ are given by:

$$
\begin{aligned}
& \text { (i) } \ddot{h}\left(q^{*}, \dot{q}^{*}\right)<0 \\
& \text { (ii) }|d h(q) \dot{q}|<\min \left\{\bar{c}_{1}, \bar{c}_{2}, \bar{c}_{3}\right\},
\end{aligned}
$$

where $\quad \bar{c}_{1}=\frac{a_{\min }\left(1-e^{\prime}\right)}{2 e} \epsilon_{t}$

$$
\begin{aligned}
& \bar{c}_{2}=\frac{a_{\min }\left(1-e^{\prime}\right)}{2 e \beta} \epsilon_{q}^{\prime} \\
& \bar{c}_{3}=\epsilon_{v}^{\prime} /\left(\frac{(1+e) \eta}{1-e^{\prime \prime}}+\frac{2 e \zeta}{a_{\min }\left(1-e^{\prime}\right)}+\frac{1}{\left\|d h\left(q^{*}\right)\right\|}\right),
\end{aligned}
$$

$\epsilon_{q}^{\prime}, \epsilon_{v}^{\prime}, a_{\min }$ and $a_{\max }$ satisfy (9) and $e^{\prime}, e^{\prime \prime}, \beta, \eta$ and $\zeta$ are defined in (10).

Note that the first condition in (14) is precisely the condition for local stability of the Zeno equilibrium point $\left(q^{*}, \dot{q}^{*}\right)$. The second condition in (14) requires that the collision velocity $\dot{h}\left(q\left(\tau_{k}\right)\right)$ is sufficiently small. The following theorem states that the reliable truncation conditions guarantee the desired bounds on the error between the exact Zeno point $\left(q_{\infty}, \dot{q}_{\infty}\right)$ and the truncated and re-initialized state $\left(q^{*}, \dot{q}^{*}\right)$, as well as on the exact Zeno time.

Theorem 3: Let $\chi^{\mathscr{H}_{\mathrm{L}}}$ be an execution of a simple Lagrangian hybrid system $\mathscr{H}_{\mathbf{L}}$. Then if there exists $k \in \Lambda$ such that $\left(q_{k-1}\left(\tau_{k}\right), \dot{q}_{k-1}\left(\tau_{k}\right)\right)$ satisfy the reliable truncation conditions with respect to given $\epsilon_{q}, \epsilon_{v}$, and $\epsilon_{t}$, then $\chi^{\mathscr{H}_{\mathbf{L}}}$ is a Zeno execution, whose Zeno time satisfies $\tau_{\infty}-\tau_{k}<\epsilon_{t}$, and its Zeno point satisfies $\left\|q_{\infty}-q^{*}\right\|<\epsilon_{q}$ and $\left\|\dot{q}_{\infty}-\dot{q}^{*}\right\|<$ $\epsilon_{v}$, where $\left(q^{*}, \dot{q}^{*}\right)=R^{*}\left(q_{k-1}\left(\tau_{k}\right), \dot{q}_{k-1}\left(\tau_{k}\right)\right)$.

Using this theorem, one can reliably truncate a Zeno execution at a time $\tau_{k}$ after a finite number $k$ of steps with guaranteed error bounds.

Proof: First, condition (i) in (14) implies that $\left(q^{*}, \dot{q}^{*}\right)$ is a stable Zeno equilibrium point, and that the neighborhood $V=N\left(\epsilon_{q}^{\prime}, \epsilon_{v}^{\prime}\right)$ exists. Choosing $\delta_{q}^{\prime}=0$ and $\delta_{v}^{\prime}=$ $d h\left(q^{*}\right) \dot{q} /\left\|d h\left(q^{*}\right)\right\|$, and defining the neighborhood $W^{\prime}$ of 
$\left(q^{*}, \dot{q}^{*}\right)$ as in (11), the definition of $R^{*}$, along with condition (ii) in (14) imply that $\left(q_{k-1}\left(\tau_{k}\right), \dot{q}_{k-1}\left(\tau_{k}\right)\right) \in W^{\prime}$. We can then exploit the time-invariance of $\mathscr{H}_{\mathbf{L}}$ to shift the time $\tau_{k}$ to $\tau_{1}$, and treat the remaining part of the execution $\chi^{\mathscr{H}_{\mathrm{L}}}$ of times $t \geq \tau_{k}$ (i.e. past the truncation point) as an execution in the vicinity of $\left(q^{*}, \dot{q}^{*}\right)$, having initial conditions within $W^{\prime}$. Lemma 2 then implies the desired bounds on the Zeno point and the Zeno time of $\chi^{\mathscr{H}_{\mathrm{L}}}$, where the definitions (15) are straightforward substitution of the chosen $\delta_{q}^{\prime}$ and $\delta_{v}^{\prime}$ into (13).

Procedure for the practical simulation of a completed Lagrangian hybrid system We now present the procedure for practical simulation of a completed Lagrangian hybrid system $\overline{\mathscr{H}}_{\mathbf{L}}$, under desired bounds $\epsilon_{q}, \epsilon_{v}, \epsilon_{t}$ on the truncation errors.

1) Initialization: set an initial time $\tau_{0}$ and initial conditions $\left(q\left(\tau_{0}\right), \dot{q}\left(\tau_{0}\right)\right)$.

2) If $h\left(q\left(\tau_{0}\right)\right)=0$ and $d h\left(q\left(\tau_{0}\right)\right) \dot{q}\left(\tau_{0}\right)=0$, go to step 5 .

3) Simulate an execution of the hybrid system $\mathscr{H}_{\mathbf{L}}$, until it reaches a collision at time $\tau_{k}$ such that $\left(q\left(\tau_{k}\right), \dot{q}\left(\tau_{k}\right)\right)$ satisfy the reliable truncation conditions.

4) Set $\tau_{0}=\tau_{k}$ and $\left(q\left(\tau_{0}\right), \dot{q}\left(\tau_{0}\right)\right)=R^{*}\left(q\left(\tau_{k}\right), \dot{q}\left(\tau_{k}\right)\right)$.

5) Simulate the constrained dynamical system $\mathscr{D}_{\mathbf{L}}$ until it reaches a time $t^{\prime}$ at which $\lambda\left(q\left(t^{\prime}\right), \dot{q}\left(t^{\prime}\right)\right)=0$.

6) Set $\tau_{0}=t^{\prime}$ and $\left(q\left(\tau_{0}\right), \dot{q}\left(\tau_{0}\right)\right)=\left(q\left(t^{\prime}\right), \dot{q}\left(t^{\prime}\right)\right)$.

7) Return to step 3.

Remarks: It is important to discuss the ramifications of the proposed procedure, which will be done through a series of remarks. First, note that in some cases, step 2 or step 5 may never terminate. This happens when either the hybrid execution is not Zeno and extends to infinite time, or when the constrained execution is consistent, i.e. satisfies $\lambda>0$, for infinite time.

Second, note that in practice, the quantities $a_{\min }, a_{\max }, \eta$, $\beta$ and $\zeta$ need not be computed exactly. Instead, one can use simplified conservative approximations of them, e.g. $\tilde{a}_{\text {min }}<$ $a_{\min }, \tilde{a}_{\max }>a_{\max }, \tilde{\beta}>\beta$, et cetera.

Finally, note that Theorem 3 only implies that the bounds on the truncation error hold for a single truncation, and not for the overall cumulative error of a long-time simulation with multiple truncations. Other sources of numerical errors in simulation of hybrid executions are numerical integration errors during the continuous phases of constrained and unconstrained motion, as well as inaccuracies in the detection of zero-crossing events $h(q(t))=0$ and in the re-initialized state. While discussion of numerical integration errors is beyond the scope of this paper, the problem of event detection and re-initialization inaccuracies can be partially solved by defining an alternative set of coordinates $q^{\prime}$ such that $h(q)$ is one of the new coordinates. This enables easier detection of the event $h=0$, and allows for manually enforcing $h=0$ at the post-collision configuration. Moreover, these coordinates are also useful for numerical integration of the constrained dynamics $\mathscr{D}_{\mathbf{L}}$ with higher accuracy, by enforcing $h=\dot{h}=0$ at each time step.

\section{Simulation Results}

This section presents numerical simulation results of the completed hybrid systems for the two running examples in this paper: the ball bouncing on a sinusoidal surface and the double pendulum with a mechanical stop.

Example 3 (Ball): Continuing with Example 1, by direct computation the condition for stability of a Zeno equilibrium point $(q, \dot{q})$ in this system as given in Theorem 2 is:

$$
\ddot{h}(q, \dot{q})=\dot{x}^{2} \sin (x)-g<0 .
$$

with $q=(x, y)$. This indicates that the points on the surface of Zeno equilibria, $Z$, that satisfy $\sin (x)>0$ (i.e. near the maxima) can attract Zeno executions only if the initial horizontal velocity $\dot{x}$ is sufficiently small.

For this example, we chose the numerical parameters $m=1$ and $g=1$, and a coefficient of restitution $e=$ 0.5 . Figures 4(a),(b),(c) show time plots of $x(t), y(t)$ and $h(q(t))$, respectively, for initial conditions $q(0)=(0,2)$ and $\dot{q}(0)=(1.5,0)$. Solutions of constrained executions appear as solid curves, while solutions of hybrid executions appear as dashed curves. The points of collision events are marked with squares ('ø'). Figure 4(e) plots $x(t)$ vs. $y(t)$, with the constraint surface $y=\sin (x)$ appearing as a thin solid line. The results of this representative simulation show initial bouncing of the ball which converges to a Zeno equilibrium point at approximately $t=3$. Then the dynamics switches to a constrained motion until the contact force $\lambda(q, \dot{q})$ vanishes at approximately $t=4$. The dynamics switches back to a hybrid execution, which converges to another Zeno point at approximately $t=11$. Finally, switching again to the constrained dynamics, the solution is then "trapped" near a minimum, and exhibits an undamped pendulum-like periodic motion for infinite time, since the constrained dynamics (5) does not include any dissipation terms.

Example 4 (Double Pendulum): In the second running example (Example 2), consisting of a double pendulum with a mechanical stop, the condition for stability of Zeno equilibria given in Theorem 2 is

$$
\ddot{h}(q, \dot{q})=\frac{g \sin \theta_{1}}{\tilde{L}}<0 \text {, where } \tilde{L}=\frac{\left(4 m_{1}+3 m_{2}\right) L_{1} L_{2}}{3\left(m_{1}\left(L_{1}+2 L_{2}\right) m_{2} L_{2}\right)} .
$$

This indicates that only states in $Z$ at which $\sin \left(\theta_{1}\right)<0$ (i.e. the link $L_{1}$ is inclined to the left) are stable Zeno equilibria.

For this example, we chose the numerical parameters $m_{1}=m_{2}=L_{1}=L_{2}=g=1$, and a coefficient of restitution $e=0.5$. Figure 4(e),(f) show the time plots of $\theta_{1}(t)$ and $\theta_{2}(t)$ under initial conditions $q(0)=\left(30^{\circ}, 25^{\circ}\right)$ and $\dot{q}(0)=(0,0)$. Again, solutions of constrained executions appear as solid curves, while solutions of hybrid executions appear as dashed curves. The results show a cyclic-like periodic motion, at which link 2 repeatedly hits the mechanical stop and bounces, until it converges to a Zeno point at which $\theta_{1}<0$ and establishes contact. Then the two links attach and swing as a single rigid pendulum in a constrained motion. When $\theta_{1}$ crosses zero, the constraining force $\lambda$ vanishes, and the two links separate again, as predicted by the stability condition. At this point the dynamics switches back to the 

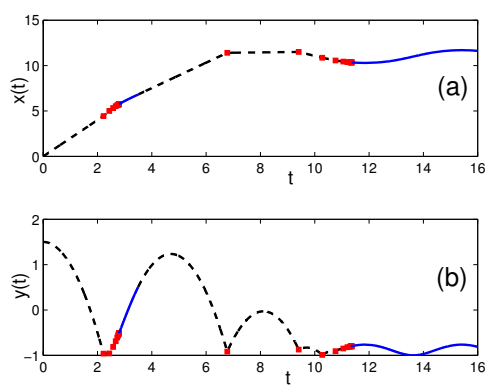
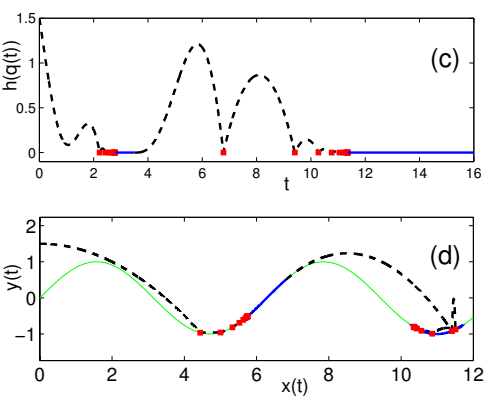
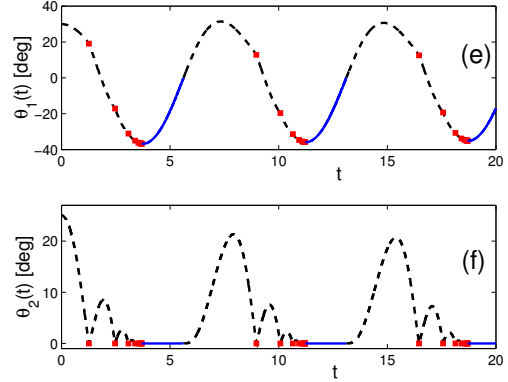

Fig. 4. Simulation results for the ball ((a)-(d)) on a sinusoidal surface and the double pendulum with a mechanical stop ((e)-(f)).

hybrid system, and the solution exhibits a sequence of collision events and converges again to a Zeno equilibrium point at $\theta_{1} \approx-40^{\circ}$. One can notice that this periodic-like motion is actually decaying, due to the energy dissipation induced by the collisions. A possible way for achieving energypreserving periodic trajectories for this mechanical system is to compensate for the energy losses by adding a controlled torque at the base of the pendulum, as demonstrated in our recent work [14].

\section{CONCLUding Discussion}

This paper presented a formal method for completion of simple Lagrangian hybrid systems by augmenting Zeno executions with solutions of the holonomically constrained system associated with the unilateral constraint. The paper also provided a method for practical simulation of the completed system such that the Zeno behavior of the exact system is preserved with guaranteed bounds on the approximation error. We now briefly discuss some high-level implications and future extensions of the results.

First, note that the practical completion procedure described in this paper essentially approximates a completed Lagrangian hybrid system by transforming it into a hybrid system with two domains, where the conditions of reliable truncation play the role of a (rather complicated) guard, and the re-initialization map $R^{*}$ is the reset map that sends the solution to the constrained surface $h^{-1}(0)$, which is the second domain. This approximation of the completed system, which cannot be exactly simulated in practice, enables practical simulation and further numerical investigation.

Second, the stability of a Zeno equilibrium point $\left(q^{*}, \dot{q}^{*}\right)$ can also be viewed as stability of the unilaterally constrained motion on the surface $h^{-1}(0)$ under small perturbations that violate the constraint, with guaranteed finite-time convergence back to the constraint surface via a Zeno hybrid execution with an infinite number of collisions. The equivalence of the conditions $\ddot{h}\left(q^{*}, \dot{q}^{*}\right)<0$ and $\lambda\left(q^{*}, \dot{q}^{*}\right)>0$ implies that the constrained motion is proven to be stable in that sense at a point $x^{*}=\left(q^{*}, \dot{q}^{*}\right)$ if and only if the constrained dynamical system is consistent at $x^{*}$, i.e. satisfies $\lambda\left(q^{*}, \dot{q}^{*}\right)>0$. This intuitively appealing result is currently limited to mechanical systems with a single unilateral constraint, involving frictionless constrained dynamics in the form (5), under frictionless collisions. However, the authors are not aware of any similar result in the literature.
Finally, the paper focused on simple Lagrangian hybrid systems having a single domain and a single guard. Extending the results to mechanical systems with multiple unilateral constraints will enable analysis of complex mechanical and robotic systems with intermittent contacts, such as bipedal walkers with knees (e.g. [15] and [10]), without using the unrealistic assumption of perfectly plastic impacts, see [14] for preliminary steps in this direction.

\section{REFERENCES}

[1] A. D. Ames, H. Zheng, R. D. Gregg, and S. Sastry, "Is there life after Zeno? Taking executions past the breaking (Zeno) point," in Proc. American Control Conference, 2006, pp. 2652 - 2657.

[2] B. Brogliato, Nonsmooth Mechanics. Springer-Verlag, 1999.

[3] M. K. Camlibel and J. M. Schumacher, "On the Zeno behavior of linear complementarity systems," in Proc. IEEE Conf. on Decision and Control, 2001, pp. $346-351$.

[4] A. Chatterjee and A. Ruina, "A new algebraic rigid body collision law based on impulse space considerations," Journal of Applied Mechanics, vol. 65, no. 4, pp. 939-951, 1998.

[5] A. Filippov, Differential equations with discontinuous right-hand sides. Kluwer Academic Publishers, 1988.

[6] R. Goebel and A. R. Teel, "Lyapunov characterization of Zeno behavior in hybrid systems," in Proceedings of the 47th IEEE Conference on Decision and Control, 2008, pp. 2752-2757.

[7] K. H. Johansson, M. Egerstedt, J. Lygeros, and S. Sastry, "On the regularization of Zeno hybrid automata," Systems and Control Letters, vol. 38 , pp. 141-150, 1999.

[8] A. Lamperski and A. D. Ames, "On the existence of Zeno behavior in hybrid systems with non-isolated Zeno equilibria," in Proc. IEEE Conf. on Decision and Control, 2008, pp. 2776-2781.

[9] J. Lygeros, K. H. Johansson, S. Simic, J. Zhang, and S. Sastry, "Dynamical properties of hybrid automata," IEEE Transactions on Automatic Control, vol. 48, pp. 2- 17, 2003.

[10] T. McGeer, "Passive walking with knees," in Proc. IEEE Int. Conf. on Robotics and Automation, vol. 3, 1990, pp. 1640 - 1645.

[11] B. M. Miller and J. Bentsman, "Generalized solutions in systems with active unilateral constraints," Nonlinear Analysis: Hybrid Systems, vol. 1, pp. 510-526, 2007.

[12] R. M. Murray, Z. Li, and S. Sastry, A Mathematical Introduction to Robotic Manipulation. CRC Press, 1993.

[13] Y. Or and A. D. Ames, "Stability of Zeno equlibria in Lagrangian hybrid systems," in Proc. IEEE Conf. on Decision and Control, 2008, pp. 2770-2775.

[14] _ "Existence of periodic orbits with Zeno behavior in completed Lagrangian hybrid systems," in Hybrid Systems: Computation and Control. Springer-Verlag, 2009, pp. 291-305.

[15] J. Pratt and G. A. Pratt, "Exploiting natural dynamics in the control of a planar bipedal walking robot," in Proceedings of the 36th Annual Allerton Conference on Communications, Control and Computing, 1998, pp. 739-748.

[16] J. Shen and J.-S. Pang, "Linear complementarity systems: Zeno states," SIAM Journal on Control and Optimization, vol. 44, no. 3, pp. 10401066, 2005.

[17] J. Zhang, K. H. Johansson, J. Lygeros, and S. Sastry, "Zeno hybrid systems," Int. J. Robust and Nonlinear Control, vol. 11, no. 2, pp. 435-451, 2001. 